\title{
Wind mass transfer in S-type symbiotic binaries
}

\section{Indication of wind focusing}

\author{
N. Shagatova, A. Skopal, and Z. Cariková
}

\begin{abstract}
Astronomical Institute, Slovak Academy of Sciences, 05960 Tatranská Lomnica, Slovakia e-mail: nshagatova@ta3.sk
\end{abstract}

Received 12 January 2015 / Accepted 28 January 2016

\section{ABSTRACT}

\begin{abstract}
Context. The wind mass transfer from a giant to its white dwarf companion in symbiotic binaries is not well understood. For example, the efficiency of wind mass transfer of the canonical Bondi-Hoyle accretion mechanism is too low to power the typical luminosities of the accretors. However, recent observations and modelling indicate a considerably more efficient mass transfer in symbiotic binaries. Aims. We determine the velocity profile of the wind from the giant at the near-orbital-plane region of eclipsing S-type symbiotic binaries EG And and SY Mus, and derive the corresponding spherical equivalent of the mass-loss rate. With this approach, we indicate the high mass transfer ratio.

Methods. We achieved this aim by modelling the observed column densities taking into account ionization of the wind of the giant, whose velocity profile is derived using the inversion of Abel's integral operator for the hydrogen column density function.

Results. Our analysis revealed the spherical equivalent of the mass-loss rate from the giant to be a few times $10^{-6} M_{\odot} \mathrm{yr}^{-1}$, which is a factor of $\gtrsim 10$ higher than rates determined by methods that do not depend on the line of sight. This discrepancy rules out the usual assumption that the wind is spherically symmetric. As our values were derived from near-orbital-plane column densities, these values can be a result of focusing the wind from the giant towards the orbital plane.

Conclusions. Our findings suggests that the wind from giants in S-type symbiotic stars is not spherically symmetric, since it is enhanced at the orbital plane and, thus, is accreted more effectively onto the hot component.
\end{abstract}

Key words. binaries: symbiotic - stars: mass-loss - stars: winds, outflows

\section{Introduction}

Symbiotic stars are binaries with the most distant components interacting via a wind mass transfer (e.g. Mürset \& Schmid 1999). Usually, the donor is a red giant (RG) and the accretor is a white dwarf (WD). The process of accretion heats the WD up to $\gtrsim 10^{5} \mathrm{~K}$ and makes it as luminous as a few times $10-10^{3} L_{\odot}$. As a result, the hot WD ionizes a large fraction of the wind from the giant, giving rise to the symbiotic nebula. The so-called S-type systems comprise a normal RG producing a stellar type of the infrared (IR) continuum, while D-type symbiotics contain a Mira-type variable, which produces an additional strong emission from the dust in the IR (Webster \& Allen 1975).

The wind accretion process represents the principal interaction between the binary components; this interaction is responsible for the appearance of the symbiotic phenomenon. However, already in the 1970s and 80s, when the binary nature of these objects was unambiguously confirmed by the International Ultraviolet Explorer (IUE), an unsolved problem arised: "How do RGs, well within their Roche lobes, lose sufficient mass to produce the symbiotic phenomenon?" (Kenyon $\&$ Gallagher 1983). The problem inheres in the large measured energetic output from hot components, which requires accretion at $10^{-8}-10^{-7} M_{\odot} \mathrm{yr}^{-1}$, and mass-loss rates of $\approx 10^{-8}$, from M-type giants, that are too low (e.g. Reimers 1977). Values of $\approx 10^{-7} M_{\odot} \mathrm{yr}^{-1}$, as derived for RGs in S-type symbiotic stars from radio observations (e.g. Seaquist et al. 1993; Mikołajewska et al. 2002), do not adequately explain the problem owing to a very low mass transfer efficiency (equivalent to the mass transfer ratio, i.e. the mass accretion rate divided by the mass-loss rate) of a few percent. Such a low mass transfer efficiency is achieved by a standard Bondi-Hoyle wind accretion (Bondi \& Hoyle 1944; Livio \& Warner 1984). Even more sophisticated threedimensional hydrodynamic simulations showed that the mass transfer ratio runs from 0.6 to $10 \%$, depending on the binary configuration (e.g. Theuns et al. 1996; Walder 1997; Walder et al. 2008). Nagae et al. (2004) found that the mass accretion rate can be even smaller than that expected via the simple Bondi-Hoyle approach. On the other hand, these authors also recognized a significant increase of the mass transfer ratio for very slow winds on the Roche surface of the mass-losing star.

Recent hydrodynamical simulations suggested a very efficient wind mass transfer for slow and dense winds, which is typical for asymptotic giant branch stars. In this mass transfer mode, called wind Roche-lobe overflow (WRLOF; Mohamed \& Podsiadlowski 2007, 2012) or gravitational focusing (de Val-Borro et al. 2009), the wind of the evolved star is focused towards the orbital plane and in particular towards the WD, which then accretes at a significantly larger rate than from the spherically symmetric wind. This mode of wind mass transfer was simulated for Mira-type binaries, and thus can be in effect in D-type symbiotic stars. An effective wind mass transfer can also be caused by a rotation of the mass-losing star. Recently, Skopal \& Cariková (2015) applied the wind compression disk model (see Bjorkman \& Cassinelli 1993; Ignace et al. 1996) to slowly rotating normal giants in S-type symbiotic stars, and found that their winds can be focused at the equatorial plane with a factor of 5-10 relative to the spherically symmetric wind. 
Table 1. Column densities of neutral hydrogen $n_{\mathrm{H}^{0}}^{\text {obs }}$ we measured on UV IUE and HST(STIS) spectra of EG And (Sect. 2).

\begin{tabular}{cccc}
\hline \hline MJD & $\varphi$ & $n_{\mathrm{H}^{0}}^{\text {obs }}\left[\mathrm{cm}^{-2}\right]$ & Spectrum \\
\hline 52630.37 & 0.0348 & $(6.0+14.0 /-5.0) \times 10^{25}$ & O8C406040 \\
49261.70 & 0.0543 & $(1.4+0.6 /-0.5) \times 10^{24}$ & SWP48820 \\
55058.75 & 0.0666 & $(2.2+0.7 /-0.7) \times 10^{23}$ & OB7503020 \\
49270.60 & 0.0727 & $(1.4+0.5 /-0.4) \times 10^{23}$ & SWP48883 \\
49270.64 & 0.0728 & $(1.4+0.5 /-0.4) \times 10^{23}$ & SWP48884 \\
55062.81 & 0.0750 & $(1.2+0.3 /-0.3) \times 10^{23}$ & OB7504020 \\
44450.68 & 0.0847 & $(1.5+0.7 /-0.5) \times 10^{23}$ & SWP09644 \\
49277.58 & 0.0872 & $(9.0+4.0 /-4.0) \times 10^{22}$ & SWP48938 \\
49277.65 & 0.0874 & $(9.0+4.0 /-4.0) \times 10^{22}$ & SWP48939 \\
49291.69 & 0.1165 & $(4.0+2.0 /-2.0) \times 10^{22}$ & SWP49056 \\
55106.42 & 0.1654 & $(4.0+2.0 /-1.5) \times 10^{20}$ & OB7505010 \\
55108.42 & 0.1695 & $(3.0+1.0 /-1.0) \times 10^{20}$ & OB7506020 \\
\hline
\end{tabular}

Finally, an efficient mass transfer was also evidenced observationally for symbiotic stars Mira AB (Karovska et al. 2005), SS Leporis (Blind et al. 2011; Boffin et al. 2014a), and FG Serpentis (Boffin et al. 2014b).

In this contribution, we introduce another indication that the winds from giants in two eclipsing S-type symbiotic stars, EG And and SY Mus, can be focused towards the equatorial plane. We analyse the measured column densities of neutral hydrogen as a function of the orbital phase following the method of Knill et al. (1993). We improve the application of this method by calculating the ionization structure of the wind. In Sect. 2, we determine column densities of neutral hydrogen around the inferior conjunction of the RG, and in Sect. 3, we derive the corresponding theoretical function. The fitting of this function to the observed quantities and resulting parameters are introduced in Sect. 4. Discussion of results and conclusion can be found in Sects. 5 and 6.

\section{Column density from Rayleigh scattering}

In the simplest ionization model of a symbiotic binary, the neutral region usually has the shape of a cone with the giant below its top facing the WD (Seaquist et al. 1984, hereafter STB). This implies that the column density of neutral hydrogen on the line of sight to the WD, $n_{\mathrm{H}^{0}}^{\mathrm{obs}}$, is a function of the orbital phase $\varphi$ and inclination $i$ of the binary $(\varphi=0$ at the inferior conjunction of the giant).

The most indicative presence of the neutral hydrogen in symbiotic binaries is a strong depression of the continuum around the hydrogen lines of the Lyman series caused by the Rayleigh scattering (see Fig. 1 of Nussbaumer et al. 1989). Assuming it is the dominant process attenuating the WD radiation, we can write the observed flux $F_{\lambda}\left(\varphi_{j}\right)$ in the $j$ th direction as

$F_{\lambda}\left(\varphi_{j}\right)=F_{\lambda}^{0} \mathrm{e}^{-n_{\mathrm{H}^{0}}^{\mathrm{obs}}\left(\varphi_{j}\right) \sigma_{\mathrm{Ray}}(\lambda)}$

where $j=1, \ldots, N, N$ is the number of $n_{\mathrm{H}^{0}}^{\mathrm{obs}}\left(\varphi_{j}\right)$ values at $\varphi_{j}$, $F_{\lambda}^{0}$ is the original (unabsorbed) flux, and $\sigma_{\text {Ray }}(\lambda)$ is the Rayleigh scattering cross section (see Eq. (5) and Fig. 2 of Nussbaumer et al. 1989). For the sake of simplicity, and because of a very high WD temperature of $\sim 10^{5} \mathrm{~K}$, we approximated the far-ultraviolet (far-UV) continuum with the black-body radiation. Fitting the function (1) to the observed continuum then yields the value of $n_{\mathrm{H}^{0}}^{\text {obs }}$ at the given phase. This approach was used and described in more detail by, for example Isliker et al. (1989), Vogel (1991), and Dumm et al. (1999).

\subsection{Column densities for EG And and SY Mus}

According to physical and orbital parameters of EG And and SY Mus (Vogel 1992; Schmutz et al. 1994; Harries \& Howarth 1996; Mürset \& Schmid 1999), their giants are well inside the Roche lobes with a corresponding ratio of $R_{\mathrm{G}} / R_{\mathrm{L}} \sim 0.50$. Therefore, we can assume that the mass transfer in EG And and SY Mus is proceeding via the wind from their RGs. A suggestion that giants in these systems fill around $85 \%$ of their Roche lobe is based on the explicit assumption that the wave-like variation in their light curves is caused by the geometrical effect of the tidal distortion of the surface of the giant (see Wilson \& Vaccaro 1997; Rutkowski et al. 2007).

Both systems are eclipsing, showing a deep atmospheric eclipse effect caused by the Rayleigh attenuation of the farUV continuum (Vogel 1990; Pereira 1996; Dumm et al. 1999; Pereira et al. 1999). Vogel (1992) estimated the inclination of the EG And orbit $i=78.5^{\circ}-90^{\circ}$ with a confidence of $72 \%$. Therefore, we consider that $i>70^{\circ}$. For SY Mus, Harries \& Howarth (1996) determined $i=84.2 \pm 1.7^{\circ}$ from its spectropolarimetric orbit.

In fitting the observed continuum (1), we approximated the flux emitted by the WD with that of a black body radiating at temperatures of $95000 \mathrm{~K}$ and $110000 \mathrm{~K}$ for EG And (Skopal 2005) and SY Mus (Mürset et al. 1991), respectively. For EG And, we determined new values of $n_{\mathrm{H}^{0}}^{\mathrm{obs}}$ using the IUE spectra from 1980-1993 and the Hubble Space Telescope (HST) spectra obtained in 2002 and 2009 (Table 1). EG And spectra were dereddened with $E_{B-V}=0.05$ mag (Mürset et al. 1991) and SY Mus spectra were dereddened with $E_{B-V}=0.35 \mathrm{mag}$ (Skopal 2005). We adopted other $n_{\mathrm{H}^{0}}^{\text {obs }}$ values from Crowley et al. (2008). The IUE spectra from the ingress part of the UV light curve (i.e. obtained prior to the inferior conjunction of the giant) were too noisy to be used. Therefore, we only modelled the data from the egress, i.e. after the giant conjunction. Quantities of $n_{\mathrm{H}^{0}}^{\text {obs }}$ for SY Mus were adopted from Dumm et al. (1999). They cover both the ingress and egress part of the light curve. We also justified the adopted $n_{\mathrm{H}^{0}}^{\text {obs }}$ values for both stars and found a good agreement with our results. Our estimate of uncertainties was $\approx 30-50 \%$, which is comparable with that of Dumm et al. (1999). Figure 1 shows examples of the Rayleigh attenuation models at different orbital phases, while Fig. 2 summarizes all $n_{\mathrm{H}^{0}}^{\text {obs }}$ values for EG And. For EG And and SY Mus, we used the ephemeris for the inferior conjunction of the giant according to Fekel et al. (2000) and Dumm et al. (1999), respectively.

\section{The method}

\subsection{Theoretical column densities}

Throughout this paper, we consider a generally used assumption that the wind is spherically symmetric. However, our results (Table 3) contradict this requirement. Thereby, we use the proof by contradiction to demonstrate the presence of wind focusing (Sect. 5.3).

In the spherically symmmetric model of the wind, its continuity equation can be written as

$\dot{M}=4 \pi r^{2} N_{\mathrm{H}}(r) \mu m_{\mathrm{H}} v(r)$,

where $\dot{M}$ is the mass-loss rate from the giant, $r$ the distance from its centre, $N_{\mathrm{H}}(r)$ the total hydrogen number density, $\mu$ the mean molecular weight, $m_{\mathrm{H}}$ the mass of the hydrogen atom, and $v(r)$ the velocity of the wind. If we integrate $N_{\mathrm{H}}(r)$ given by Eq. (2) along the line-of-sight $l$ from the observer $(-\infty)$ to the infinity 
N. Shagatova et al.: Wind mass transfer in S-type symbiotic binaries. II.
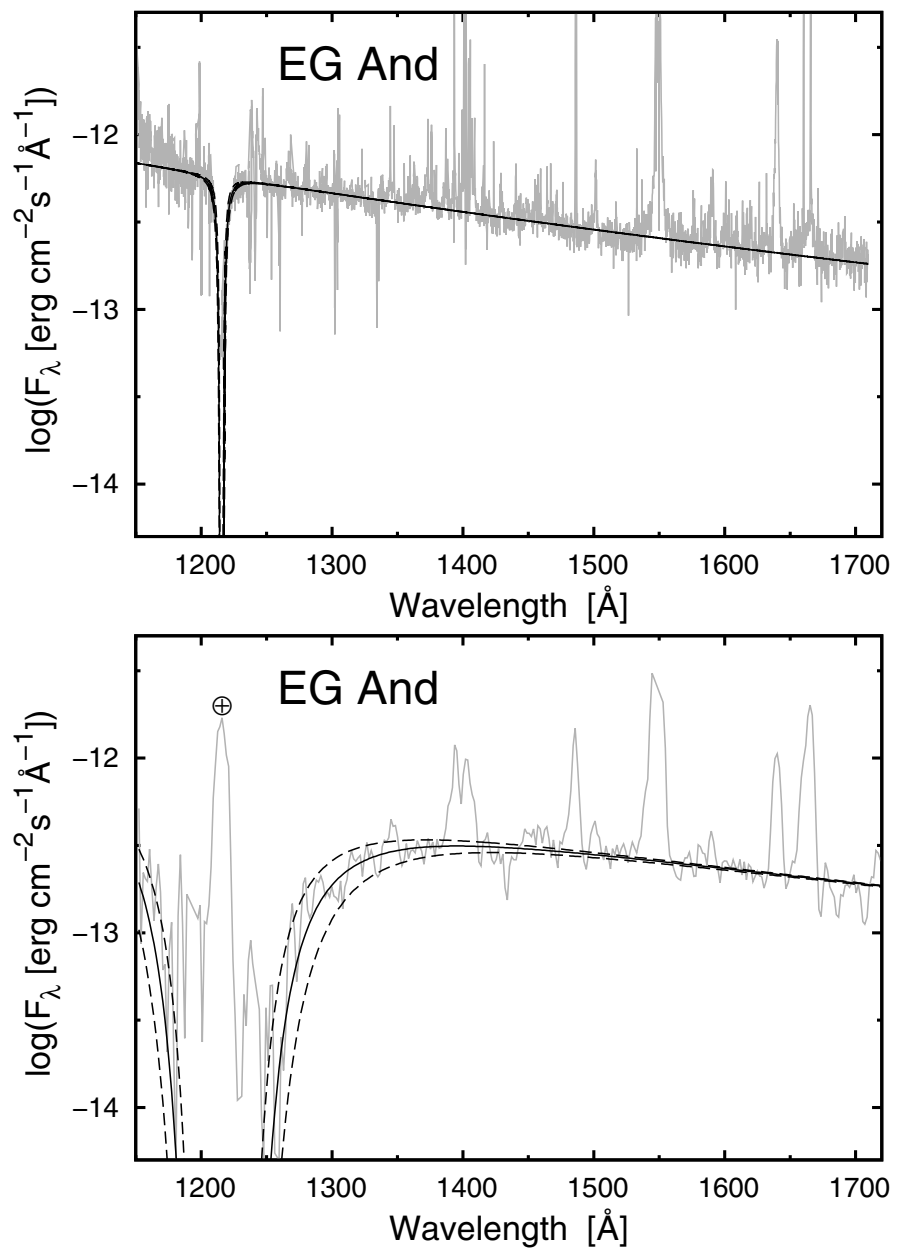

Fig. 1. Examples of the far-UV spectrum of EG And (grey line) compared to models (solid and dashed lines) given by Eq. (1) at different orbital phases. Top: $\varphi=0.1695, n_{\mathrm{H}^{0}}^{\mathrm{obs}}=(3 \pm 1) \times 10^{20} \mathrm{~cm}^{-2}$. Bottom: $\varphi=0.0847, n_{\mathrm{H}^{0}}^{\mathrm{obs}}=(1.5+0.7 /-0.5) \times 10^{23} \mathrm{~cm}^{-2}($ see Table 1$) . \oplus$ denotes the geocoronal Ly- $\alpha$ emission.

containing the WD, the column density of total hydrogen, $n_{\mathrm{H}}$, can be expressed as

$n_{\mathrm{H}}=\frac{\dot{M}}{4 \pi \mu m_{\mathrm{H}}} \int_{-\infty}^{\infty} \frac{\mathrm{d} l}{r^{2} v(r)}$.

Using the separation between the binary components $p$, projected to the plane perpendicular to the line of sight, the so-called impact parameter $b$ (e.g. Vogel 1991),

$b^{2}=p^{2}\left(\cos ^{2} i+\sin ^{2} \varphi \sin ^{2} i\right)$,

and the relation $r^{2}=l^{2}+b^{2}$ (e.g. Fig. 1 of Knill et al. 1993), we can rewrite Eq. (3) to the form

$n_{\mathrm{H}}(b)=a \int_{b}^{\infty} \frac{\mathrm{d} r}{r v(r) \sqrt{r^{2}-b^{2}}}$,

where

$a=\frac{2 \dot{M}}{4 \pi \mu m_{\mathrm{H}}}$

If the wind velocity profile (WVP), $v(r)$, is known, we can directly calculate $n_{\mathrm{H}}$ at a given phase, since the distances $l$ and $r$

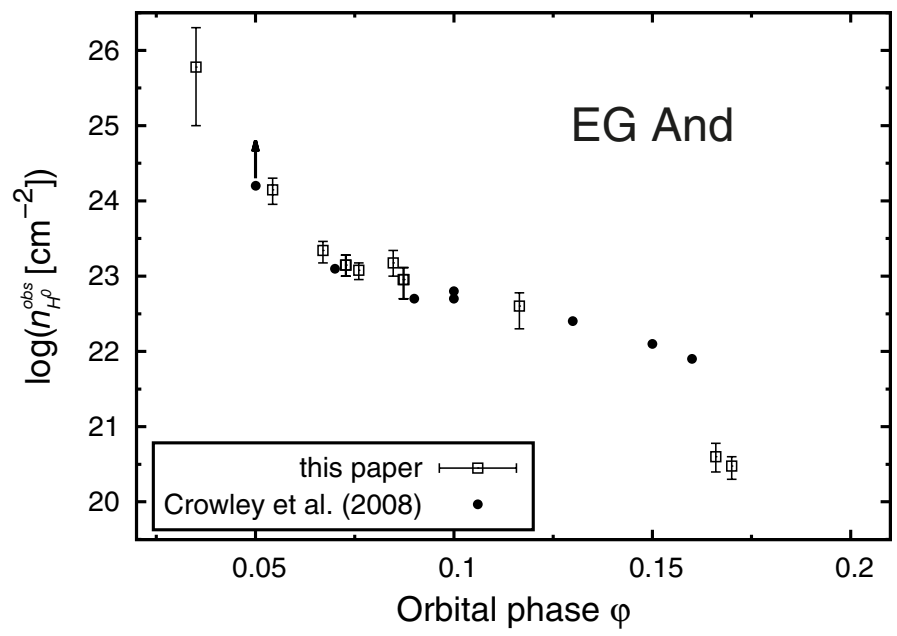

Fig. 2. Egress column densities as a function of the orbital phase for EG And. Our values (Table 1) and those of Crowley et al. (2008) are depicted by squares and circles, respectively.

depend on $\varphi$. However, the situation is just the opposite; we know $n_{\mathrm{H}}$ from observations, but the WVP is unknown. So, we have an inversion problem, which was solved by Knill et al. (1993).

\subsection{The wind velocity profile formula}

Basically, the method of Knill et al. (1993) allows us to determine the WVP with the inversion of the integral operator for column density by its diagonalization. Since this inversion problem is generally unstable, the diagonalization is restricted to the finite series with an appropriate number of terms. According to Dumm et al. (1999), the adequate finite sum representing the column density in the inversion method can be expressed as

$\tilde{n}_{\mathrm{H}}(b)=\frac{n_{1}}{b}+\frac{n_{K}}{b^{K}}$,

where coefficients $n_{1}, n_{K}$, and $K$ can be obtained by fitting the observed $n_{\mathrm{H}^{0}}^{\mathrm{obs}}$ values (see Sect. 4.1). The first term dominates for large values of $b$, while the second term dominates for small values of $b$. It is not necessary to use the $\tilde{n}_{\mathrm{H}}(b)$ function with more terms because of the large errors in the $n_{\mathrm{H}^{0}}^{\text {obs }}$ data (Table 1). Moreover, Eq. (7) represents a good approximation of the original $n_{\mathrm{H}}(b)$ function (5) and has a simple inverted form,

$\frac{a}{r v(r)}=\frac{n_{1}}{\lambda_{1} r}+\frac{n_{K}}{\lambda_{K} r^{K}}$

For $r \rightarrow \infty, \frac{a}{r v(r)} \rightarrow \frac{n_{1}}{\lambda_{1} r}$ and $v(r) \rightarrow v_{\infty}$, where $v_{\infty}$ is the terminal velocity of the wind, we obtain the relation

$\frac{a}{v_{\infty}}=\frac{n_{1}}{\lambda_{1}}$

and finally, using Eq. (8), the WVP has the form

$v(r)=\frac{v_{\infty}}{1+\xi r^{1-K}}$,

where $\xi=\frac{n_{K} \lambda_{1}}{n_{1} \lambda_{K}}, \lambda_{1}$, and $\lambda_{K}$ are the eigenvalues of the Abel operator given by the relation (Knill et al. 1993)

$$
\begin{array}{ll}
i=1 & \lambda_{1}=\frac{\pi}{2}, \\
i \geq 2 & \lambda_{i}=\frac{\pi}{2(i-1) \lambda_{i-1}} .
\end{array}
$$


Equation (10) is sufficiently flexible to represent a great variety of velocity profiles (e.g. to be comparable to the widely used $\beta$-law; see Fig. A.2).

To summarize, the parameters $n_{1}, n_{K}$, and $K$ can be obtained by fitting the measured $n_{\mathrm{H}^{0}}^{\mathrm{obs}}$ data with Eq. (7), and thus the WVP in the form of Eq. (10). However, since the giant wind is partially ionized, we consider this effect in our approach.

\subsection{Effect of the ionization on $H^{0}$ column density}

During quiescent phases, a fraction of the neutral wind from the giant is ionized by the hard radiation emitted by the hot component. Hence, the shape of the neutral $\mathrm{H}^{0}$ region is determined by the $\mathrm{H}^{0} / \mathrm{H}^{+}$boundary. Therefore, to calculate a value of $\mathrm{H}^{0}$ column density, we have to integrate Eq. (3) from the observer to the intersection of the line of sight with the $\mathrm{H}^{0} / \mathrm{H}^{+}$boundary, $l(\varphi)$ (see Fig. 2 of Skopal \& Shagatova 2012), i.e.

$n_{\mathrm{H}^{0}}=\frac{a}{2} \int_{-\infty}^{l_{\varphi}} \frac{\mathrm{d} l}{r^{2} v(r)}$.

Using the parameter $b$ (Eq. (4)), which determines the direction of the line-of-sight $l$ to the WD, Eq. (12) can be rewritten as

$n_{\mathrm{H}^{0}}(b)=\frac{a}{2} \int_{-\infty}^{l_{\varphi}(b)} \frac{\mathrm{d} l}{\left(l^{2}+b^{2}\right) v\left(\sqrt{l^{2}+b^{2}}\right)}$,

which we use to model the $n_{\mathrm{H}^{0}}^{\text {obs }}$ data (Sect. 4).

Finally, the $\mathrm{H}^{0} / \mathrm{H}^{+}$interface can be obtained from the condition of the ionization/recombination equilibrium between the flux of ionizing photons from the WD and rate of recombinations in the wind from the giant. According to Nussbaumer \& Vogel (1987), the solution of the parametric equation

$f(u, \vartheta)-X^{\mathrm{H}+}=0$,

defines the boundary between neutral and ionized gas at the orbital plane determined by a system of polar coordinates $(u, \vartheta)$ with the origin at the hot star. The ionization parameter $X^{\mathrm{H}+}$ is written as

$X^{\mathrm{H}+}=\frac{4 \pi\left(\mu m_{\mathrm{H}}\right)^{2}}{\alpha_{\mathrm{B}}\left(\mathrm{H}, T_{\mathrm{e}}\right)} p L_{\mathrm{ph}}\left(\frac{v_{\infty}}{\dot{M}}\right)^{2}$,

where $L_{\mathrm{ph}}$ is the flux of ionizing photons from the WD and $\alpha_{\mathrm{B}}\left(\mathrm{H}, T_{\mathrm{e}}\right)$ is the total hydrogen recombination coefficient for recombinations other than to the ground state (Case B) at the electron temperature, $T_{\mathrm{e}}$. The function $f(u, \vartheta) \propto v(r)^{-2}$ was treated for the first time by STB for a steady state situation and a constant wind velocity. Examples of the $\mathrm{H}^{0} / \mathrm{H}^{+}$boundaries for different WVP can be found in, for example Nussbaumer \& Vogel (1987), Skopal (2001), and Skopal \& Shagatova (2012). Intersection of the line of sight with the boundary is determined by the parameter $l_{\varphi}(b)$ (see Sect. 3.3 of Skopal \& Shagatova 2012).

As a result of a very rapid transition of wind hydrogen from an almost completely neutral to an almost fully ionized state at the ionization boundary, the flux of ionizing photons penetrating the neutral region is negligible and the flux of the neutral hydrogen atoms to the ionized zone is even of less importance (see e.g. Figs. 2c and 6 of Schwank et al. 1997, or Fig. 4.14 of Crowley 2006).

\section{Modelling and results}

\subsection{The procedure}

In this section, we determined the WVP and the corresponding $\dot{M}$ for giants in EG And and SY Mus by fitting the measured column densities $n_{\mathrm{H}^{0}}^{\text {obs }}$ with the function (13). This indicates we need to find parameters $n_{1}, n_{K}$ and $K$, which determine the WVP in the form of Eq. (10) and parameter $X^{\mathrm{H}+}$, which determines the geometry of the $\mathrm{H}^{0} / \mathrm{H}^{+}$boundary. To avoid unreasonably high values of $n_{1}$ and $n_{K}$, we used the giant radius, $R_{\mathrm{G}}$, as the length unit. First, we estimated possible ranges of fitting parameters with a preliminary match of the data with the function (13). Then, we proceeded as follows:

(i) Using the Monte Carlo method, we assigned values of all searched parameters, $n_{1}, n_{K}, K$, and $X^{\mathrm{H}+}$, within the given ranges.

(ii) For the corresponding WVP and the $X^{\mathrm{H}+}$ parameter, we solved Eq. (14) to get the intersection of the line of sight with the ionization boundary determined by the parameter $l_{\varphi}(b)$.

(iii) Then, we calculated the $n_{\mathrm{H}^{0}}(b)$ function (Eq. (13)) for the model WVP and $l_{\varphi}(b)$, compared this function with the $n_{\mathrm{H}^{0}}^{\text {obs }}$ data, and obtained the reduced $\chi$-squared sum, $\chi_{\text {red }}^{2}$.

(iv) Finally, iterating this procedure within the space of input parameters provided us a set of models. We selected the best solution using the condition of the least-squares method.

In the case of SY Mus, the best fits were found empirically because of scanty data for $b<2.5$ (see Fig. 4). To match the ingress data, we used relation (see Eq. (15))

$X^{\mathrm{H}+} \propto\left(\frac{v_{\infty}}{\dot{M}}\right)^{2}$,

to obtain the ingress/egress $n_{1}$ parameters ratio (see Eq. (18) below), and thus the ingress $n_{1}$ value for the model egress $n_{1}$ parameter.

\subsection{The spherical equivalent of the mass-loss rate}

The inversion method allows us to determine the value of the corresponding mass-loss rate in the spherically symmetric model, i.e. the spherical equivalent of the mass-loss rate $\dot{M}_{\mathrm{sp}}$. By analogy to Eq. (9) for the integral (5), we can write

$\frac{a}{2 R_{\mathrm{G}}}=\frac{n_{1} v_{\infty}}{\lambda_{1}}$

for the integral (13), expressed in the length units of $R_{\mathrm{G}}$, for $r \rightarrow \infty$. Then, using Eq. (6) we obtain the relationship

$\dot{M}_{\mathrm{sp}}=4 \pi \mu m_{\mathrm{H}} R_{\mathrm{G}} \frac{n_{1}}{\lambda_{1}} v_{\infty}$,

where $n_{1}$ is the resulting parameter of our models (see Table 2).

Comparison of $\dot{M}_{\text {sp }}$ quantities (Table 3) with the total massloss rates, $\dot{M}$, determined by methods that do not depend on the line of sight (Sect. 5.1), allows us to test whether the wind from giants in S-type symbiotic binaries is spherically symmetric or not (Sect. 5.3).

\subsection{Estimate of uncertainties}

The primary source of uncertainties of the fitting parameters is given by the errors of the measured column densities $n_{\mathrm{H}^{0}}^{\text {obs }}$ 
N. Shagatova et al.: Wind mass transfer in S-type symbiotic binaries. II.

Table 2. Resulting parameters $\left(n_{1}, n_{\mathrm{K}}, K\right.$ and $\left.X^{\mathrm{H}+}\right)$ of modelling $n_{\mathrm{H}^{0}}^{\mathrm{obs}}$ column densities (Sect. 2.1) with the function (13).

\begin{tabular}{lccccccccc}
\hline \hline Object & $i$ & $\mathrm{E} / \mathrm{I}^{a}$ & $n_{1}\left[10^{23}\right]$ & $n_{\mathrm{K}}$ & $K$ & $\xi$ & $X^{\mathrm{H}+}$ & $\chi_{\text {red }}^{2}$ & Model \\
\hline EG And & $70^{\circ}$ & $\mathrm{E}$ & 4.54 & $5.12 \times 10^{30}$ & 21 & $6.40 \times 10^{7}$ & 1.75 & 1.67 & $\mathrm{I}$ \\
& $80^{\circ}$ & $\mathrm{E}$ & 3.87 & $1.15 \times 10^{27}$ & 14 & $1.38 \times 10^{4}$ & 1.85 & 1.60 & $\mathrm{~J}$ \\
& $90^{\circ}$ & $\mathrm{E}$ & 3.40 & $4.83 \times 10^{25}$ & 10 & $5.49 \times 10^{2}$ & 1.88 & 1.63 & $\mathrm{~K}$ \\
SY Mus & $80^{\circ}$ & $\mathrm{E}$ & 8.00 & $1.50 \times 10^{27}$ & 9 & $8.94 \times 10^{3}$ & 2.30 & 1.02 & $\mathrm{~L}$ \\
& $84^{\circ}$ & $\mathrm{E}$ & 6.20 & $5.30 \times 10^{26}$ & 8 & $2.94 \times 10^{3}$ & 2.50 & 0.94 & $\mathrm{M}$ \\
& $90^{\circ}$ & $\mathrm{E}$ & 6.10 & $5.00 \times 10^{26}$ & 8 & $2.82 \times 10^{3}$ & 2.53 & 1.39 & $\mathrm{~N}$ \\
& $84^{\circ}$ & $\mathrm{I}$ & 2.45 & $1.00 \times 10^{27}$ & 13 & $1.81 \times 10^{4}$ & 16.0 & 2.33 & $\mathrm{O}$ \\
\hline
\end{tabular}

Notes. They define the WVP in the form of Eq. (10) and the ionization structure of the wind given by the solution of Eq. (14). ${ }^{(a)}$ E - egress data, I - ingress data.

(Table 1). Therefore, to estimate possible ranges of the model parameters, $n_{1}, n_{K}, K$, and $X^{\mathrm{H}+}$, we prepared a grid of corresponding models (13) around the best solution to cover the measured column densities within their errors. In this way, we estimated uncertainties in $n_{1}$ to be in the range of $10-30 \%$, which, according to Eq. (18), can also be assigned to $\dot{M}$. We found small uncertainties around of 3-6\% for the parameter $X^{\mathrm{H}+}$ because its value is given by the orbital phase, at which the line of sight coincides with the tangent to the $\mathrm{H}^{0} / \mathrm{H}^{+}$boundary (i.e. when $n_{\mathrm{H}^{0}}^{\text {obs }} \rightarrow 0$ ). In both cases, this tangent is well defined by the smallest values of $n_{\mathrm{H}^{0}}^{\text {obs }}$ at $\varphi \sim 0.17$ and 0.16 for EG And and SY Mus. The $K$ index is always within $10 \%$ of its most probable values. However, the parameter $n_{\mathrm{K}}$ is very uncertain (>70\%) because its value dominates the $\tilde{n}_{\mathrm{H}}(b)$ function (Eq. (7)) for small $b$, where the $n_{\mathrm{H}^{0}}^{\mathrm{obs}}$ measurements suffer from large errors (Table 1).

A further source of uncertainties is given by the assumption of the spherical symmetry. Since the $\mathrm{H}^{0}$ column density data show the ingress/egress asymmetry and the main result of the wind focusing (see below Sect. 5.3) rules out the spherically symmetric wind, the WVPs derived in this paper are also affected by the following systematic error. However, it is hard to assess its magnitude. Since there is a lack of observations resolving spatial wind distribution for S-type symbiotic systems, our approach can provide, at least, some constraints on theoretical modelling of the circumstellar matter in symbiotic binaries.

Another source of the systematic error is given by using a subset of the full space of eigenfunctions of Abel operator to avoid ill-conditioning, as proposed by Knill et al. (1993). Since the column density models represent data well, we suppose this uncertainty to be of less magnitude than errors from other mentioned sources.

\subsection{Application to EG And and SY Mus}

According to the geometry of the $\mathrm{H}^{0}$ zone in the binary, as given by $n_{\mathrm{H}^{0}}^{\text {obs }}$ measurements (e.g. Fig. 2), we calculated the $n_{\mathrm{H}^{0}}(b)$ function (13) for the range of orbital phases $\langle 0.02,0.21\rangle$ and adopted orbital inclinations $i$. According to Vogel (1992) and Harries \& Howarth (1996), $i>70^{\circ}, p=330 R_{\odot}$ and $i=84^{\circ} .2 \pm 1.7, p=376 R_{\odot}$ for EG And and SY Mus, respectively. Therefore, we selected $i=70,80,90^{\circ}$ and $i=80,84,90^{\circ}$ in fitting the $n_{\mathrm{H}^{0}}^{\mathrm{obs}}$ values of EG And and SY Mus. Resulting parameters are summarized in Table 2 and the corresponding models are shown in Figs. 3 and 4. For the EG And model with $i=90^{\circ}$, the highest value of $n_{\mathrm{H}^{0}}^{\mathrm{obs}}$ has $b \lesssim 1$, which suggests that $i<90^{\circ}$ because the line of sight would pass through the giant itself. Nevertheless, we show this solution just for comparison.

To determine the spherical equivalent of the mass-loss rate from the giant according to Eq. (18), we used $R_{\mathrm{G}}=75$ and $86 R_{\odot}$
Table 3. Spherical equivalent of the mass-loss rate, $\dot{M}_{\text {sp }}$ (Eq. (18)), corresponding to model parameters in Table 2 for $v_{\infty}=30 \mathrm{~km} \mathrm{~s}^{-1}$.

\begin{tabular}{lccc}
\hline \hline Object & $i$ & $\dot{M}_{\text {sp }}\left[M_{\odot} \mathrm{yr}^{-1}\right]$ & Model \\
\hline EG And & $70^{\circ}$ & $2.11 \times 10^{-6}$ & $\mathrm{I}$ \\
& $80^{\circ}$ & $1.80 \times 10^{-6}$ & $\mathrm{~J}$ \\
& $90^{\circ}$ & $1.58 \times 10^{-6}$ & $\mathrm{~K}$ \\
SY Mus & $80^{\circ}$ & $4.26 \times 10^{-6}$ & $\mathrm{~L}$ \\
& $84^{\circ}$ & $3.30 \times 10^{-6}$ & $\mathrm{M}$ \\
& $90^{\circ}$ & $3.24 \times 10^{-6}$ & $\mathrm{~N}$ \\
& $84^{\circ}$ & $1.30 \times 10^{-6}$ & $\mathrm{O}$ \\
\hline
\end{tabular}

for EG And (Vogel 1992) and SY Mus (Schmutz et al. 1994) and characteristic $v_{\infty}=20-50 \mathrm{~km} \mathrm{~s}^{-1}$ (Sect. 5.1). Examples for $v_{\infty}=$ $30 \mathrm{~km} \mathrm{~s}^{-1}$ are introduced in Table 3 .

\section{Discussion}

\section{1. $v_{\infty}$ and $\dot{M}$ for giants in S-type symbiotic stars}

According to Dupree (1986), terminal velocities of winds from cool giants are $<100 \mathrm{~km} \mathrm{~s}^{-1}$ with decreasing values for later spectral types. For EG And, Crowley (2006) determined $v_{\infty}=$ $75 \mathrm{~km} \mathrm{~s}^{-1}$ from the radial velocity of the absorption component of the $\mathrm{Mg}^{+}$resonance doublet measured on the spectrum during the inferior conjunction of the giant. From molecular absorption band analysis, Dumm et al. (1999) referred $v_{\infty}$ to be in the range of $10-30 \mathrm{~km} \mathrm{~s}^{-1}$ for $\mathrm{M}$ giant winds. From radial velocities of the $\mathrm{H} \alpha$ absorption component, Skopal et al. (2001) derived $v_{\infty}=32 \pm 6 \mathrm{~km} \mathrm{~s}^{-1}$ for a M4.5 III giant in AX Per. Values of $v_{\infty}=20-30 \mathrm{~km} \mathrm{~s}^{-1}$ can be inferred from the position of the violet shifted component of the Raman scattered $\mathrm{O}^{5+} 1032 \AA$ line (Schmid et al. 1999).

Mass-loss rates from giants in symbiotic binaries are usually determined in the context of the STB model, in which a fraction of the spherically symmetric wind from the giant is ionized by the hot component. Then, measuring the wind nebular emission allows us to determine the corresponding mass-loss rate. In the radio, Seaquist et al. (1993) measured flux density at $3.6 \mathrm{~cm}$ for a sample of 99 symbiotic stars. Using the Wright \& Barlow (1975) relation, corrected for the shape of the neutral part of the wind as given by the parameter $X^{\mathrm{H}+}$ in the STB model, they derived $\dot{M} \gtrsim 10^{-7} M_{\odot} \mathrm{yr}^{-1}$ for RGs in S-type symbiotic stars. A similar conclusion was reached by Mikołajewska et al. (2002) based on analysis of radio emission at $1.3 \mathrm{~mm}$. In the UV/optical/near-IR, we can calculate the model emission measure, which is determined by parameters of the giant's wind. Here we use a technique of disentangling the composite 
A\&A 588, A83 (2016)
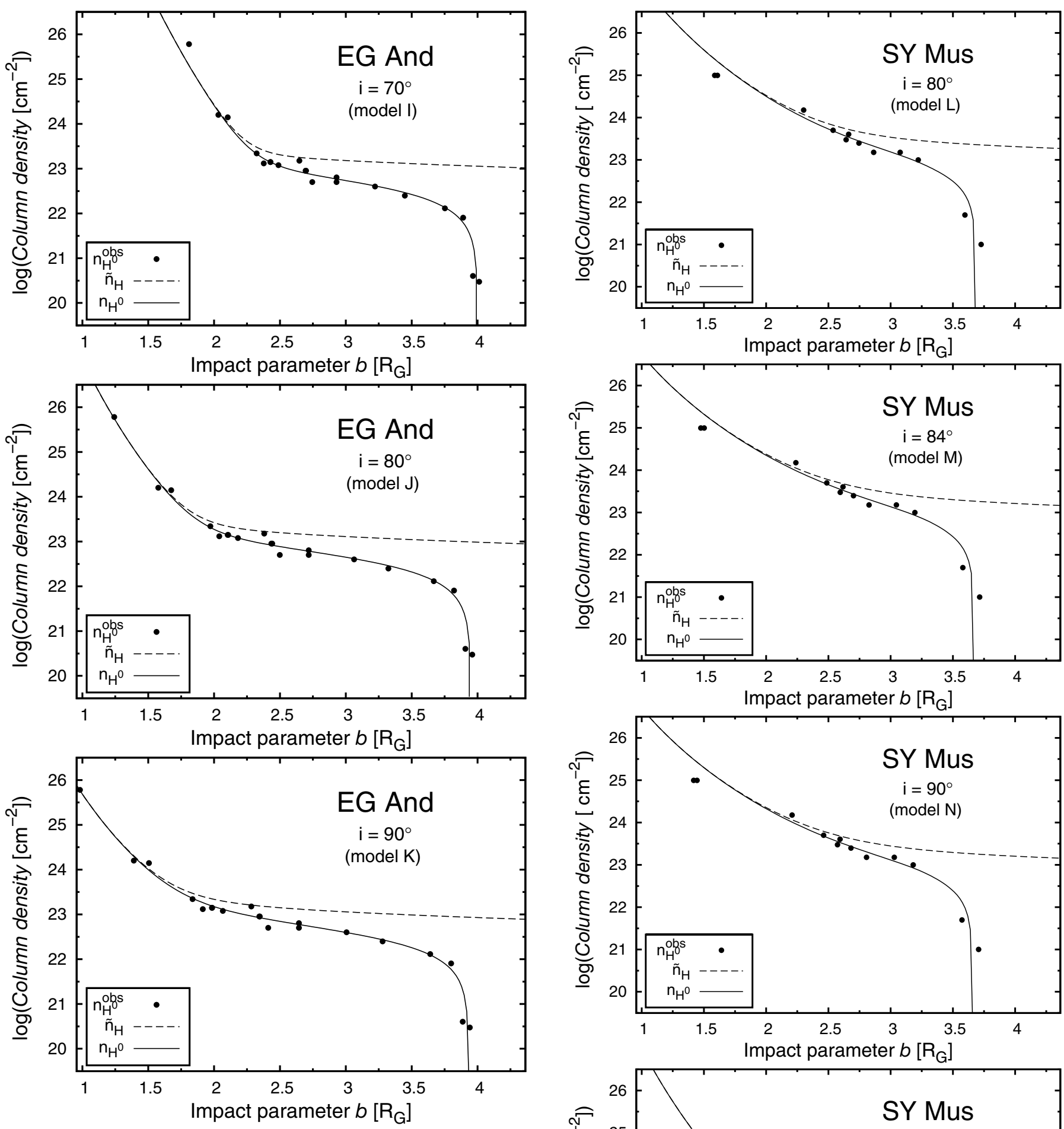

Fig. 3. Resulting fits of $n_{\mathrm{H}^{0}}^{\mathrm{obs}}\left(b_{j}\right)$ data for EG And (dots) with the $n_{\mathrm{H}^{0}}(b)$ function (Eq. (13); solid lines). Parameters of models are listed in Table 2. Corresponding $\tilde{n}_{\mathrm{H}}(b)$ functions, which represent the total hydrogen column densities (i.e. no ionization included, Eq. (7)) are depicted with dashed lines.

continuum of symbiotic binaries to extract the nebular component from the observed spectrum. In this way, Skopal (2005) determined that $\dot{M}$ for giants in 15 well-observed, S-type symbiotic stars is a few $\times 10^{-7} M_{\odot} \mathrm{yr}^{-1}$. Another possibility is provided by the parameter $X^{\mathrm{H}+}$, whose definition (see Eq. (15)) allows us to determine $\dot{M}$ for a given set of binary parameters. In this way, Mürset et al. (1991) estimated typical values of

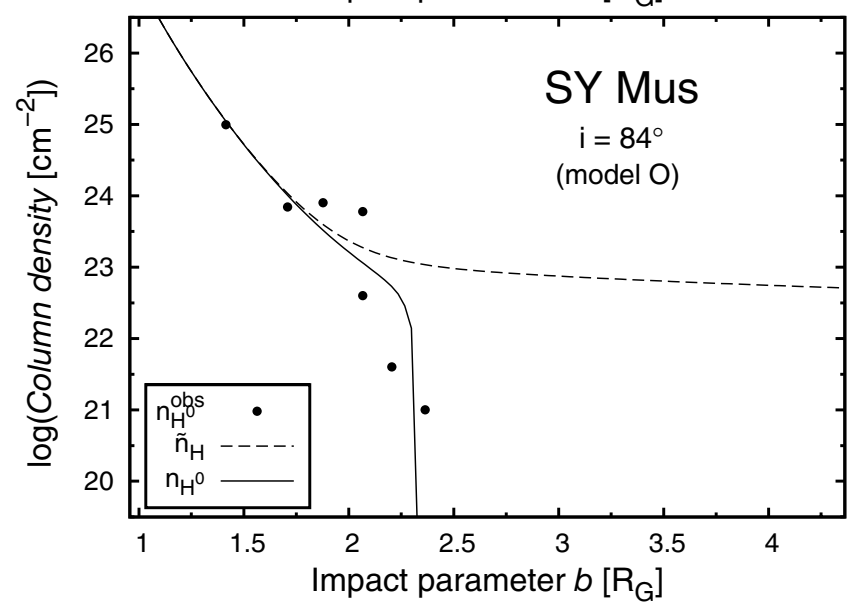

Fig. 4. As in Fig. 3, but for SY Mus data. 
N. Shagatova et al.: Wind mass transfer in S-type symbiotic binaries. II.
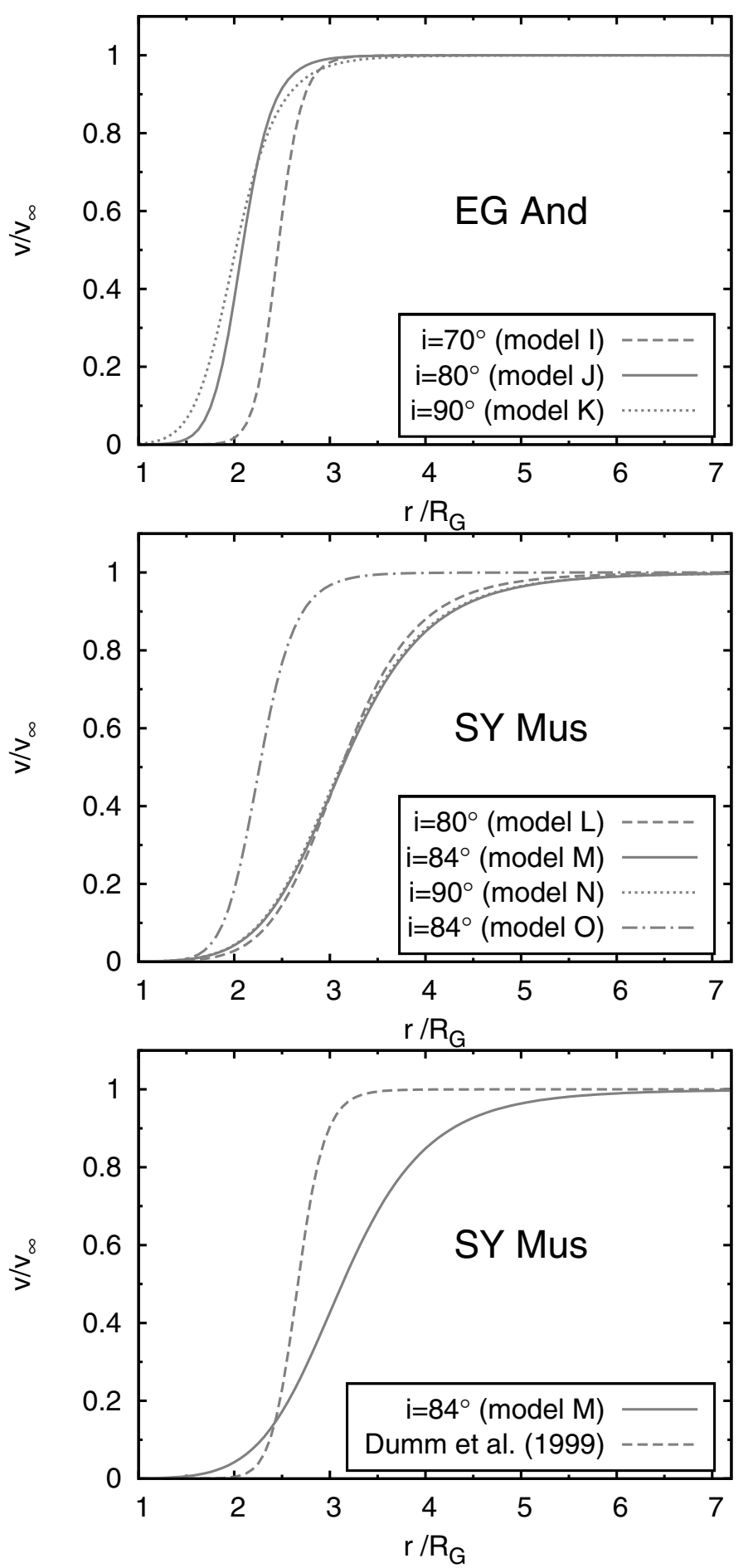

Fig. 5. WVPs of our $n_{\mathrm{H}^{0}}(b)$ models (Table 2). The bottom panel compares our model for SY Mus with that of Dumm et al. (1999), which does not calculate ionization of the wind (Sect. 5.2).

$\dot{M} \approx 10^{-7} M_{\odot} \mathrm{yr}^{-1}$ for the RGs in their sample of 12 S-type symbiotic stars.

With respect to our approach in this paper, it is important to stress that these values of $\dot{M}$ represent the total mass-loss rates, which are independent of the line of sight (see STB; Wright \& Barlow 1975).

\subsection{Effect of ionization to $W V P$ and $\dot{M}$}

Since both Dumm et al. (1999) and this work applied the same method to the same data to determine the WVP of the giant in SY Mus; we can test the effect of the wind ionization by comparing the corresponding velocity profiles.

The bottom panel of Fig. 5 compares the WVP of the giant in SY Mus derived from the column density model of Dumm et al. (1999), which does not calculate ionization of the wind, and from our $n_{\mathrm{H}^{0}}(b)$ model (Eq. (13)), which includes the effect of the wind ionization. For $r \lesssim 2 R_{\mathrm{G}}$, both models require a low $v(r)$, which reflects a high column density on the lines of sight passing in the vicinity of the cool giant. However, for $r \gtrsim 2 R_{\mathrm{G}}$ the wind of the model by Dumm et al. accelerates much faster than our model. This is given by a different particle density $N_{\mathrm{H}}(r)$ along the line of sight in the $n_{\mathrm{H}}(b)$ model (Eq. (7)) and our $n_{\mathrm{H}^{0}}(b)$ model (Eq. (13)) constrained by the same value of $n_{\mathrm{H}^{0}}^{\mathrm{obs}}(b)$. This is because in the former case, we integrate the column density of total hydrogen, i.e. from the observer to $\infty$, while in the latter case, we integrate only throughout the neutral wind, i.e. the integration ends at the intersection with the $\mathrm{H}^{0} / \mathrm{H}^{+}$boundary. Since $v(r) \propto 1 / N_{\mathrm{H}}(r)$, a lower $N_{\mathrm{H}}(r)$ in the former case implies a higher $v(r)$, which is the case of the Dumm's et al. wind model. At $r \gtrsim 5 R_{\mathrm{G}}, v(r) \rightarrow v(\infty)$ in both models.

Similarly, ionization of the giant's wind also affects the corresponding model mass-loss rate. A larger $N_{\mathrm{H}}(r)$ along the line of sight in our models with respect to models without ionization corresponds to a larger $\dot{M}$ because $\dot{M} \propto N_{\mathrm{H}}(r)$. For example, our $\dot{M}_{\text {sp }}=3 \times 10^{-6} M_{\odot} \mathrm{yr}^{-1}$ for SY Mus (Table 3) is a factor of $\sim 6$ larger than the Dumm et al. (1999) value of $\sim 5 \times 10^{-7} M_{\odot} \mathrm{yr}^{-1}$. Accordingly, we can conclude that the influence of the wind ionization in determining the WVP and $\dot{M}$ is fairly significant.

\subsection{Focusing of the wind}

Since both EG And and SY Mus are high-inclination systems, our line of sight pointing to their WDs always passes through the near-orbital-plane region and, thus, the column densities and WVP, as determined by our method, reflect characteristics of this area. We used the continuity Eq. (2) to derive the column density models given by Eqs. (7) and (13). However, the $n_{\mathrm{H}^{0}}(b)$ models correspond to the spherical equivalent of the mass-loss rate, $\dot{M}_{\text {sp }} \gtrsim 10^{-6} M_{\odot} \mathrm{yr}^{-1}$ (Table 3 ), which is a factor $\gtrsim 10$ higher than the total mass-loss rate from giants in S-type symbiotic stars (see Sect. 5.1). As the column densities were measured at the nearorbital-plane region (eclipsing binaries) and the corresponding $\dot{M}_{\text {sp }} \gg \dot{M}_{\text {total }}$, the wind from the giant has to be focused towards the orbital plane.

This result is consistent with a 3D hydrodynamic simulations of the circumstellar mass distribution in the symbiotic binary RS Oph, which show a mass enhancement in the orbital plane as compared to the polewards directions (see Walder et al. 2008). Also, focusing of the wind from normal giants in Stype symbiotic binaries towards the orbital plane can be caused by their slow rotation, as suggested by Skopal \& Cariková (2015). However, simplifying assumptions in the model by Skopal \& Cariková (2015) cannot describe the column density distribution of the circumstellar material around the giant in detail in the same way as we measure in the real case (see Appendix A). 


\section{Conclusion}

We modelled hydrogen column densities $n_{\mathrm{H}^{0}}^{\text {obs }}$ of neutral wind from RGs in eclipsing S-type symbiotic stars EG And and SY Mus with the aim at determining the WVP and the corresponding $\dot{M}$. We determined WVPs of giants in the form of Eq. (10), obtained by inversion of the Abel's integral operator for the hydrogen column density function (Eq. (3)), derived under the assumption of the spherically symmetric wind (Eq. (2)). In our approach, we fitted $n_{\mathrm{H}^{0}}^{\text {obs }}$ values by the function $n_{\mathrm{H}^{0}}(b)$ (Eq. (13)), which integrates the column density only within the neutral fraction of the giant's wind. This represents the main novelty with respect to previous analyses, in which the effect of the wind ionization was not calculated (e.g. Dumm et al. 1999).

Our approach revealed that the spherical equivalent of the mass-loss rate from giants in EG And and SY Mus $\dot{M}_{\mathrm{sp}} \gtrsim$ $10^{-6} M_{\odot} \mathrm{yr}^{-1}$ (Sect. 4.2, Table 3), which is a factor of $\gtrsim 10$ higher than total rates, $\dot{M}$, determined by techniques that do not depend on the line of sight (Sect. 5.1). This finding suggests that the wind from giants in S-type symbiotic stars is not spherically symmetric. Because the measured column densities correspond to those at the near-orbital-plane region (eclipsing binaries) and $\dot{M}_{\text {sp }} \gg \dot{M}_{\text {total }}$, the wind is focused towards the binary orbital plane. As a result, the enhanced wind at the orbital plane allows the WD to accrete from the giant's wind more effectively than in the spherically symmetric case.

Acknowledgements. The authors would like to thank the referee Ken Gayley for suggestions that helped to substantially improve this paper. This article was created by the realization of the project ITMS No. 26220120009, based on the supporting operational Research and Development Programme financed by the European Regional Development Fund. This research was supported by a grant of the Slovak Academy of Sciences, VEGA No. 2/0002/13.

\section{References}

Bjorkman, J. E., Cassinelli, J. P. 1993, ApJ, 409, 429

Blind, N., Boffin, H. M. J., Berger, J.-P., et al. 2011, A\&A, 536, A55

Boffin, H. M. J., Blind, N., Hillen, M., et al. 2014a, The Messenger, 156, 35

Boffin, H. M. J., Hillen, M., Berger, J. P. et al. 2014b, A\&A, 564, A1

Bondi, H., \& Hoyle, F. 1944, MNRAS, 114, 195

Crowley, C. 2006, Ph.D. Thesis, Trinity College Dublin, Ireland

Crowley, C., Espey, B. R., \& McCandliss, S. R. 2008, ApJ, 675, 711 de Val-Borro, M., Karovska, M., \& Sasselov, D. 2009, ApJ, 700, 1148

Dumm, T., Schmutz, W., Schild, H., \& Nussbaumer, H. 1999, A\&A 349, 169

Dupree, A. K. 1986, ARA\&A 24, 377

Espey, B. R., \& Crowley, C. 2008, in RS Ophiuchi (2006) and the Recurrent Nova Phenomenon (San Francisco: ASP), eds. A. Evans, M. F. Bode, T. J. O’Brien, M. J. Darnley, ASP Conf. Ser. 401, 166

Fekel, F. C., Joyce, R. R., Hinkle, K. H., \& Skrutskie, M. 2000, AJ, 119, 1375

Harries, T. J., \& Howarth, I. D. 1996, A\&A, 310, 235

Ignace, R., Cassinelli, J. P., \& Bjorkman, J. E. 1996, ApJ, 459, 671

Isliker, H., Nussbaumer, H., \& Vogel, M. 1989, A\&A, 219, 271

Karovska, M., Schlegel, E., Hack, W., Raymond, J. C., \& Wood, B. E. 2005, ApJ, 623, L137

Kenyon, S. J., \& Gallagher, J. S. 1983, AJ, 88, 666

Knill, O., Dgani, R., \& Vogel, M. 1993, A\&A, 274, 1002

Lamers, H. J. G. L. M., \& Cassinelli, J. P. 1999, Introduction to stellar winds (Cambridge University Press)

Livio, M., \& Warner, B. 1984, The Observatory, 104, 152

Mikołajewska, J., Ivison, R. J., \& Omont, A. 2002, Adv. Space Res., 30, 2045

Mohamed, S., \& Podsiadlowski, Ph. 2007, in 15th European Workshop on White Dwarfs, eds. R. Napiwotzki, \& M. R. Burleigh, ASP Conf. Ser., 372, 397

Mohamed, S., \& Podsiadlowski, Ph. 2012, Balt. Astron. 21, 88

Mürset, U., \& Schmid, H. M. 1999, A\&AS, 137, 473

Mürset, U., Nussbaumer, H., Schmid, H. M., \& Vogel, M. 1991, A\&A, 248, 458

Nagae, T., Oka, K., Matsuda, T., et al. 2004, A\&A, 419, 335

Nussbaumer, H., \& Vogel, M. 1987, A\&A 182, 51

Nussbaumer, H., Schmid, H. M., \& Vogel, M. 1989, A\&A 211, L27

Pereira, C. B. 1996, Ap\&SS, 235, 305

Pereira, C. B., Ortega, V. G., \& Monte-Lima, I. 1999, A\&A, 344, 607

Reimers, D. 1977, A\&A, 61, 217

Rutkowski, A., Mikołajewska, J., \& Whitelock, P. A. 2007, Balt. Astron., 16, 49

Schmid, H. M., Krautter, J., Appenzeller, I., et al. 1999, A\&A, 348, 950

Schmutz, W., Schild, H., Mürset, U., \& Schmid, H. M. 1994, A\&A, 288, 819

Schwank, M., Schmutz, W., \& Nussbaumer, H. 1997, A\&A, 319, 166

Seaquist, E. R., Taylor, A. R., \& Button, S. 1984, ApJ, 284, 202 (STB)

Seaquist, E. R., Krogulec, M., \& Taylor, A. R. 1993, ApJ, 410, 260

Skopal, A. 2001, A\&A, 366, 157

Skopal, A. 2005, A\&A, 440, 995

Skopal, A., \& Cariková, Z. 2015, A\&A, 573, A8 (Paper I)

Skopal, A., \& Shagatova, N. 2012, A\&A, 547, A45

Skopal, A., Teodorani, M., Errico, L., et al. 2001, A\&A, 367, 199

Theuns, T., Boffin, H. M. J., \& Jorissen, A. 1996, MNRAS, 280, 1264

Vogel, M. 1990, in Evolution in Astrophysics, ed. E. J. Rolfe, ESA SP-310, 393

Vogel, M. 1991, A\&A 249, 173

Vogel, M. 1992, A\&A, 260, 156

Walder, R. 1997, in Accretion phenomena and related outflows, IAU Colloq., 163, eds. D. T. Wichramasinghe, L. Ferrario, \& G. V. Bicknell, ASP Conf. Ser., 121, 822

Walder, R., Folini, D., \& Shore, S. N. 2008, A\&A, 484, L9

Webster, B. L., \& Allen, D. A. 1975, MNRAS, 171, 171

Wilson, R. E., \& Vaccaro, T. R. 1997, MNRAS 291, 54

Wright, A. E., \& Barlow, M. J. 1975, MNRAS, 170, 41 


\section{Appendix A: Comparison with the wind compression model}

Recently, Skopal \& Cariková (2015, Paper I) applied the wind compression zone model (WCZ; see Bjorkman \& Cassinelli 1993; Ignace et al. 1996) to the stellar wind of slowly rotating RGs in S-type symbiotic binaries. In this way, they showed that the high wind-mass transfer efficiency in these systems, as required by the high luminosities of their hot components, can be caused by the wind focusing towards the orbital plane.

Here, we calculate the $n_{\mathrm{H}}(b)$ and $n_{\mathrm{H}^{0}}(b)$ functions using the WCZ models A-H of Paper I (see Table 2 therein), and compare these functions with our solution using Eqs. (7) and (13), respectively. However, to judge the comparison, one has to take into account that the WCZ models of Paper I represent only a stationary case including just the kinematic of the giant's wind with a $\beta$-law velocity profile. On the other hand, the measured $n_{\mathrm{H}^{0}}^{\mathrm{obs}}(b)$ values result from the real density distribution of the circumstellar material around RGs, the nature of which is not included in the WCZ model. Therefore, we do not claim to fit the $n_{\mathrm{H}^{0}}^{\mathrm{obs}}(b)$ measurements with the WCZ model, but present just a comparison to learn more about a possible origin of their differences.

\section{A.1. Density distribution in the wind compression model}

The rotation of a star can lead to the compression of its wind towards the equatorial regions. The distribution of its density as a function of the radial distance $r$ from the RG's centre and the polar angle $\theta$ is expressed as

$N_{\mathrm{H}}(r, \theta)=\frac{\dot{M}}{4 \pi r^{2} \mu m_{\mathrm{H}} v_{\mathrm{r}}(r)}\left(\frac{\mathrm{d} \mu_{\mathrm{m}}}{\mathrm{d} \mu_{0}}\right)^{-1}$,

where $\dot{M}$ is the mass-loss rate from the star, $v_{\mathrm{r}}(r)$ is the radial component of the wind velocity, and the geometrical factor $\mathrm{d} \mu_{\mathrm{m}} / \mathrm{d} \mu_{0}$ describes the compression of the wind due to rotation of the star. A $\beta$-law WVP is considered in the model. Main assumptions are briefly summarized in Paper I, in detail by Lamers \& Cassinelli (1999), and originally formulated by Bjorkman \& Cassinelli (1993).

\section{A.2. Ionization boundaries in the WCZ model}

The $\mathrm{H}^{0} / \mathrm{H}^{+}$boundary in the WCZ model is also obtained by the solution of the parametric Eq. (14), but the parameter $X^{\mathrm{H}+}$ has its standard form,

$X^{\mathrm{H}+}=\frac{4 \pi \mu^{2} m_{\mathrm{H}}^{2}}{\alpha_{\mathrm{B}}\left(\mathrm{H}, T_{\mathrm{e}}\right)} p L_{\mathrm{ph}}\left(\frac{v_{\infty}}{\dot{M}}\right)^{2}$,

with the same denotation and meaning of parameters as in Eq. (15), and the function

$f(u, \vartheta)=\int_{0}^{u_{\varphi}(b)} \frac{u^{2}}{z^{4}\left(1-\frac{\tilde{b}}{z} \frac{R_{\mathrm{G}}}{p}\right)^{2 \beta}\left(\frac{\mathrm{d} \mu_{\mathrm{m}}}{\mathrm{d} \mu_{0}}\right)^{2}} \mathrm{~d} u$ includes the geometrical factor of the wind compression and the $\beta$-law WVP. Distances $r$ and $l$ are expressed here in units of the stars separation $p$, i.e. $z=r / p, u=l / p\left(u_{\varphi}(b)=l_{\varphi}(b) / p\right), z=$ $\sqrt{u^{2}+1-2 u \sin i \cos \vartheta}$, and $\tilde{b}=1-\left(a / v_{\infty}\right)^{1 / \beta}$ is the parameter of the wind model (see Paper I).

\section{A.3. Column densities in the WCZ model}

Using the density distribution in the WCZ model, $N_{\mathrm{H}}(r, \theta)$, the column density of the total hydrogen along the line of sight containing the WD is calculated as

$n_{\mathrm{H}}(b)=\int_{-\infty}^{\infty} N_{\mathrm{H}}(r, \theta) \mathrm{d} l$,

which is compared to Eq. (7) of this paper. Similarly, the column density restricted to the $\mathrm{H}^{0}$ zone only is calculated as

$n_{\mathrm{H}^{0}}(b)=\int_{-\infty}^{l_{\varphi}(b)} N_{\mathrm{H}}(r, \theta) \mathrm{d} l$,

which is compared to Eq. (13).

Figure A.1 provides this comparison for models $\mathrm{J}$ and $\mathrm{M}$ of this paper and WCZ models of Paper I using the input parameters in Table A.1. For $b \lesssim 2$, there is a large difference in all cases. This difference can be caused by the large uncertainties in determining of $n_{\mathrm{H}^{0}}^{\text {obs }}$ values from low-resolution spectra and by the presence of other absorbing effects (e.g. bound-free transitions and the Rayleigh attenuation in the continuum) that are not included in the model (1). For further distances $(b \gtrsim 2-2.5)$ and the column density of the total hydrogen, $n_{\mathrm{H}}(b)$ functions in the WCZ models (A.4) are comparable with $\tilde{n}_{\mathrm{H}}(b)$ (top panels of Fig. A.1). However, in models including the effect of ionization, $n_{\mathrm{H}^{0}}(b)$ functions in the WCZ models (A.5), which match the measured values within $3.5 \gtrsim b \gtrsim 2.5$, bend at larger $b$ because the $\mathrm{H}^{0}$ zone opens more at the near-orbital-plane region because of the wind compression there (bottom panels of Fig. A.1). On the other hand, the WCZ models (A.5) with the bend around $b=3.5-4$, are below the $n_{\mathrm{H}^{0}}^{\text {obs }}(b)$ values because these models (H, D, G, C for EG And and C, F, B for SY Mus, see the figure) correspond to a smaller compression, and thus to lower $N_{\mathrm{H}}(r, \theta)$ densities implying lower $n_{\mathrm{H}^{0}}(b)$ functions (A.5).

Here we note that Paper I suggests the WCZ model just as a possible efficient wind-mass transfer mode in S-type symbiotic binaries. However, simplifying assumptions of the model do not allow us to provide any information on the real structure of the wind from the rotating giant. As a result, corresponding $n_{\mathrm{H}^{0}}(b)$ functions (A.5) do not match $n_{\mathrm{H}^{0}}^{\mathrm{obs}}(b)$ values, which are given by the real density distribution in the binary. Nevertheless, the amount of the total hydrogen at the plane of observations in the WCZ models (A.4) matches that derived from the column density measurements (top panels of Fig. A.1). Finally, a good fit of the $n_{\mathrm{H}^{0}}^{\mathrm{obs}}(b)$ quantities by the function (13) is due to a very flexible WVP (10), whose variables do not have clear physical meaning, however. Example $v(r)$, which matches the $\beta$-law wind of Paper I, is shown in Fig. A.2. 
Table A.1. Input parameters for WCZ models of Paper I.

\begin{tabular}{cccccccccc}
\hline \hline Object & $R_{\mathrm{G}}\left[R_{\odot}\right]$ & $p\left[R_{\odot}\right]^{a}$ & $\dot{M}\left[M_{\odot}\right]$ & $T_{\mathrm{h}}[\mathrm{K}]$ & $L_{\mathrm{h}}\left[L_{\odot}\right]^{b}$ & $a\left[\mathrm{~km} \mathrm{~s}^{-1}\right]$ & $\beta$ & $v_{\infty}\left[\mathrm{km} \mathrm{s}^{-1}\right]$ & $v_{\mathrm{rot}}\left[\mathrm{km} \mathrm{s}^{-1}\right]^{c}$ \\
\hline EG And & 75 & 330 & $2 \times 10^{-7}$ & 95000 & 77 & 1 & 2.5 & $20-50$ & $6-9.5$ \\
SY Mus & 86 & 376 & $4 \times 10^{-7}$ & 110000 & 1200 & 1 & 2.5 & $20-50$ & $6-9.5$ \\
\hline
\end{tabular}

Notes. ${ }^{(a)}$ Parameters $R_{\mathrm{G}}$ and $p$ as in Sect. $4.4 ;{ }^{(b)}$ parameters $\dot{M}, T_{\mathrm{h}}$ and $L_{\mathrm{h}}$ are from Skopal (2005), ${ }^{(c)}$ parameters of the wind, $a, \beta, v_{\infty}$, and $v_{\text {rot }}$ are adopted from Paper I.
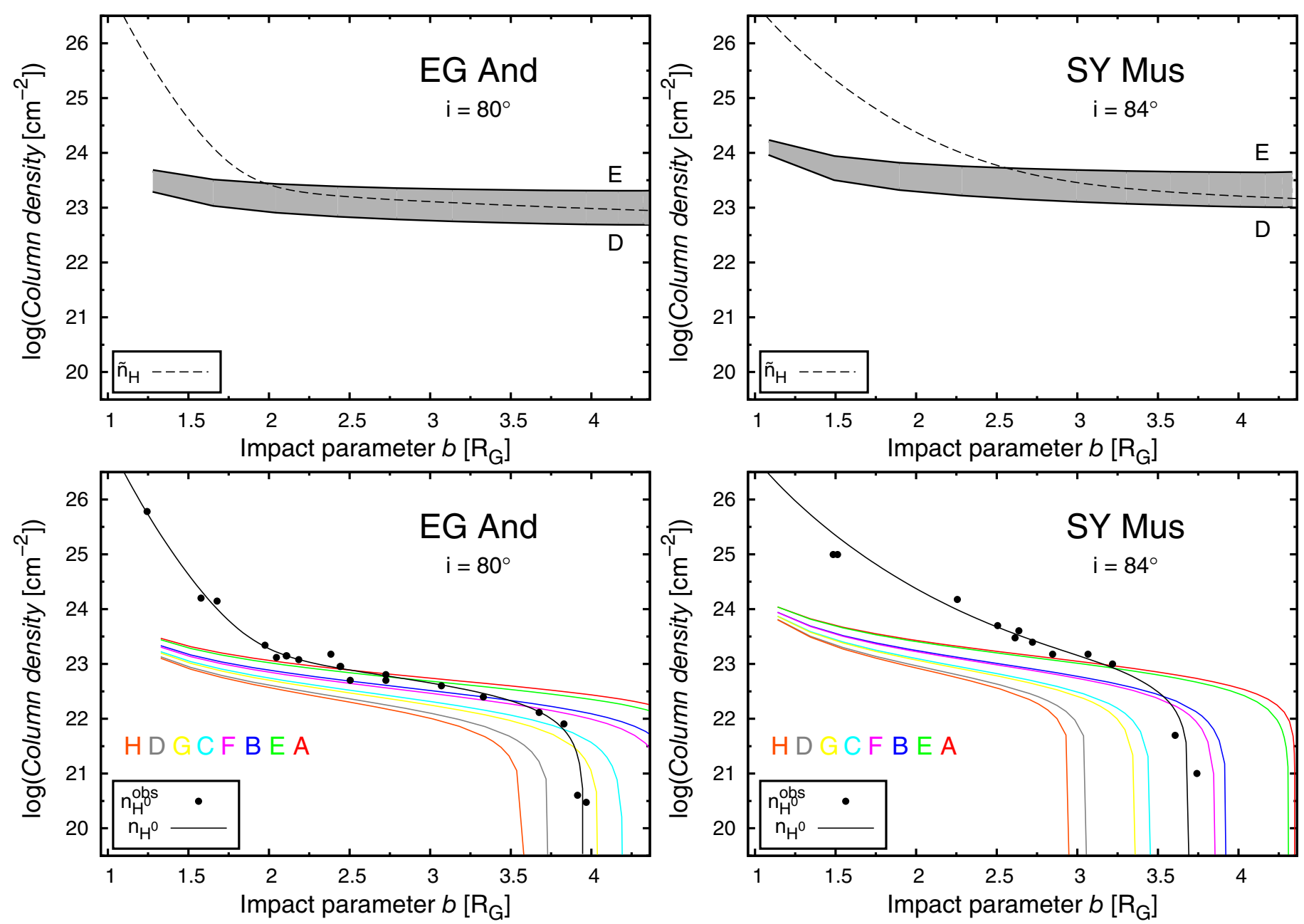

Fig. A.1. Comparison of hydrogen column densities of this paper with those calculated according to the WCZ models of Paper I. Top panels show examples of models J and M (Eq. (7); dashed lines) with those calculated according to Eq. (A.4) for WCZ models A-H of Paper I (grey area bounded by models E and D). Bottom panels compare the same models, but calculated throughout the neutral zone only (this paper: Eq. (13), solid black line; WCZ models of Paper I: Eq. (A.5), coloured lines).

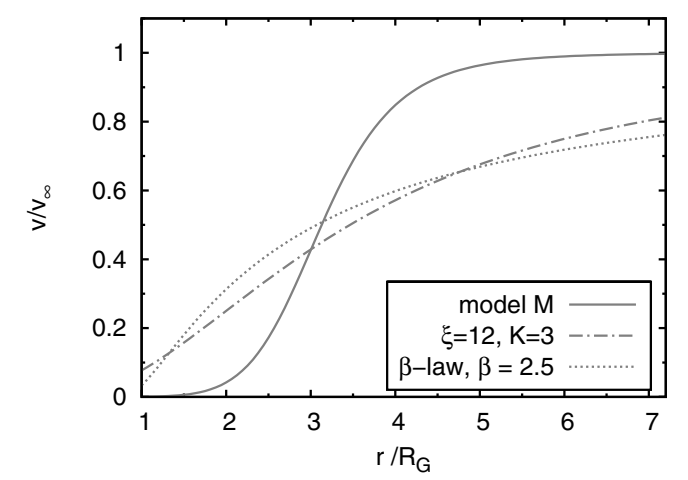

Fig. A.2. Examples of the $v(r)$ profiles and the $\beta$-law wind of Paper I. 